\title{
NeuroImage
}

\section{Cognitive priming in sung and instrumental music: Activation of inferior frontal cortex}

\author{
B. Tillmann, ${ }^{\mathrm{a}, *}$ S. Koelsch, ${ }^{\mathrm{b}}$ N. Escoffier, ${ }^{\mathrm{a}}$ E. Bigand, ${ }^{\mathrm{c}}$ P. Lalitte, ${ }^{\mathrm{c}}$ \\ A.D. Friederici, ${ }^{\mathrm{b}}$ and D.Y. von Cramon ${ }^{\mathrm{b}}$ \\ ${ }^{a}$ CNRS UMR 5020, Neurosciences et Systèmes Sensoriels, Université Claude Bernard-Lyon I, IFR 19, 50 Av. Tony Garnier, F-69366 Lyon Cedex 07, France \\ ${ }^{\mathrm{b}}$ Max-Planck-Institute of Human Cognitive and Brain Sciences, Leipzig, Germany \\ ${ }^{\mathrm{c}}$ LEAD-CNRS 5022, Université de Bourgogne, Dijon, France
}

Received 19 August 2005; revised 7 February 2006; accepted 10 February 2006

Available online 19 April 2006

\begin{abstract}
Neural correlates of the processing of musical syntax-like structures have been investigated via expectancy violation due to musically unrelated (i.e., unexpected) events in musical contexts. Previous studies reported the implication of inferior frontal cortex in musical structure processing. However - due to the strong musical manipulations activations might be explained by sensory deviance detection or repetition priming. Our present study investigated neural correlates of musical structure processing with subtle musical violations in a musical priming paradigm. Instrumental and sung sequences ended on related and less-related musical targets. The material controlled sensory priming components, and differences in target processing required listeners' knowledge on musical structures. Participants were scanned with functional Magnetic Resonance Imaging (fMRI) while performing speeded phoneme and timbre identification judgments on the targets. Behavioral results acquired in the scanner replicated the facilitation effect of related over less-related targets. The blood oxygen level-dependent (BOLD) signal linked to target processing revealed activation of right inferior frontal areas (i.e., inferior frontal gyrus, frontal operculum, anterior insula) that was stronger for less-related than for related targets, and this was independent of the material carrying the musical structures. This outcome points to the implication of inferior frontal cortex in the processing of syntactic relations also for musical material and to its role in the processing and integration of sequential information over time. In addition to inferior frontal activation, increased activation was observed in orbital gyrus, temporal
\end{abstract}

Abbreviations: BA, Brodmann area; BOLD, blood oxygen level dependent; EPI, echo planar imaging; ERAN, early right anterior negativity; ERP, event related potential; fMRI, functional magnetic resonance imaging; FWHM, full width half maximum; IFG, inferior frontal gyrus; IFS, inferior frontal sulcus; IPG, inferior parietal gyrus; IPS, intraparietal sulcus; LPC, late positivity component; mERAN, magnetic early right anterior negativity; MEG, magnetoencephalography; MFG, middle frontal gyrus; MTG, middle temporal gyrus; MIDI, Musical Instrument Digital Interface; RATN, right anterior-temporal negativity; SFG, superior frontal gyrus; SMG, supramarginal gyrus; STG, superior temporal gyrus; STS, superior temporal sulcus.

* Corresponding author. Fax: +3343728 7601 .

E-mail address: barbara.tillmann@olfac.univ-lyon1.fr (B. Tillmann).

Available online on ScienceDirect (www.sciencedirect.com). areas (anterior superior temporal gyrus, posterior superior temporal gyrus and sulcus, posterior middle temporal gyrus) and supramarginal gyrus.

(C) 2006 Elsevier Inc. All rights reserved.

Music is a complex, auditory non-verbal material, and musical syntax refers to the structural organization of musical events evolving over time (e.g., Koelsch, 2005; Patel, 2003). Neural correlates of musical structure processing have been investigated by focusing on listeners' musical expectations and notably on violations of these expectations. Inferior frontal areas (i.e., inferior frontal gyrus and adjacent anterior insula) were reported to be activated more strongly by unexpected events than by expected ones (Koelsch et al., 2002, 2005; Maess et al., 2001; Tillmann et al., 2003). Our study investigated musical syntax processing via subtle expectancy violations in instrumental and sung music. The aim was to reject alternative explanations of previously reported neural correlates in terms of repetition priming or sensory deviance processing and to focus on cognitive, knowledge-driven components of musical structure processing.

Structural regularities in Western tonal music are based on statistical regularities in the use of a restricted set of tones (i.e., the twelve tones of the chromatic scale). Three tones played simultaneously define a chord, and a subset of seven tones with their associated chords defines a key. In a musical piece, tones and chords belonging to one key are used together, with some tones and chords used more frequently than others. These differences reflect hierarchies of functional importance between tones and chords in a given key (i.e., tonal hierarchies). The tonic tone and tonic chord are at the top of these functional hierarchies and represent the most referential events of a key. The dominant and subdominant chords are, respectively, at the second and third positions of the functional hierarchy and are thus less referential than the tonic. Because of the restricted set of possible tones, the musical function of a given tone (or chord) changes with the tonal context and the currently established key: it may be out-of-key, in- 


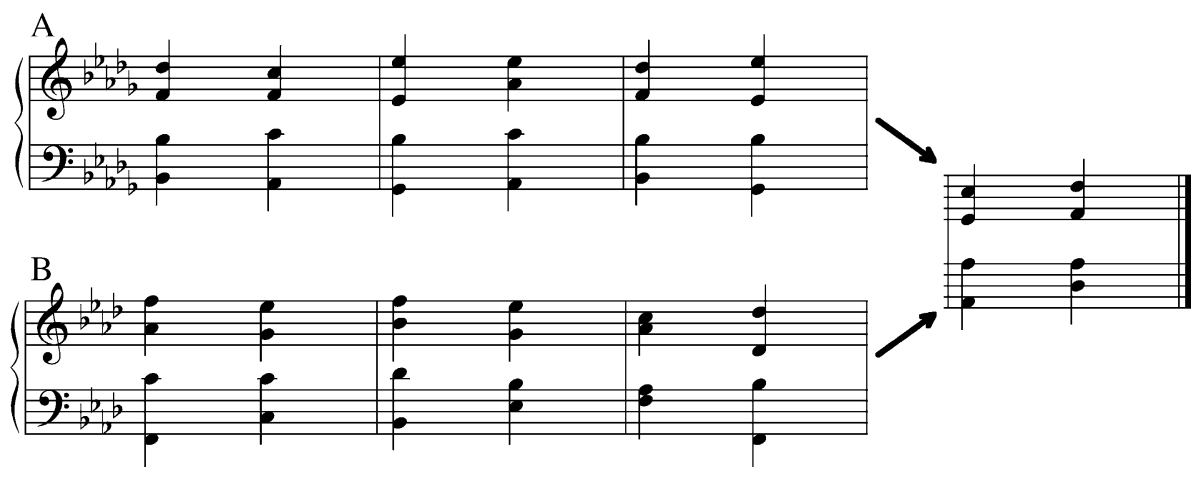

Fig. 1. Two eight-chord sequences illustrating the context effect: the final chord functions as a related tonic chord after the sequence A, and as a less-related subdominant chord after the sequence B (sound files are available at http://olfac.univ-lyon1.fr/bt-sound.html). In the priming paradigm, the first seven chords of each sequence represent the prime context and the eighth chord represents the target chord.

key or take on different levels of functional importance. For example, the same chord can function as a tonic or a subdominant depending on the context in which it occurs (Fig. 1).

Music cognition research has provided evidence that nonmusician listeners have acquired implicit knowledge about tonal regularities and that they are sensitive to the context dependency of musical events (Francès, 1958; Krumhansl, 1990; Tillmann et al., 2000). The influence of listeners' context-dependent musical expectations on event processing has been investigated with the musical priming paradigm. A prime context (e.g., a chord sequence) is followed by a to-be-processed musical target (e.g., a chord), which is either strongly related (e.g., the tonic) and thus supposed to be strongly expected, unrelated (i.e., unexpected) like an out-of-key chord, or less-related, like another in-key chord with a less important musical function (e.g., the subdominant). Target chords are processed faster after musically related contexts than after unrelated contexts (Bharucha and Stoeckig, 1987; Tillmann et al., 1998) and even after less-related contexts (Bigand and Pineau, 1997; Bigand et al., 1999, 2001). ${ }^{1}$ In musical priming, knowledge-driven processes (referred to as cognitive priming) prevail over sensory-driven processes (i.e., sensory priming). The former results from the activation of listeners' knowledge of Western musical syntax, the later from the acoustical overlap between prime and target (i.e., harmonic spectra, tone repetition). Cognitive priming is also observed for nonmusician listeners who have never received explicit musical training: the processing of related targets is facilitated even when less-related targets share more tones with the context than related targets (Bigand et al., 2003; Tekman and Bharucha, 1998) or when immediately preceded by the same chord (Bigand et al., 2005).

Neurophysiological correlates of musical structure processing have also been investigated via expectancy violations. The same tone or chord evokes a stronger late positive component LPC (peaking around 500 and $600 \mathrm{~ms}$ ) when it is unrelated to the context than when it is related (Besson and Macar, 1987; Besson et al., 1994; Janata, 1995; Patel et al., 1998). In addition to this late positive marker, which has been associated with processes of structural reanalysis or repair, earlier negative potentials have been reported for the detection of music-syntactic violations and the processing of structural dependencies. Together with source

\footnotetext{
${ }^{1}$ Participants make speeded accuracy judgments on a perceptual feature of the target without explicitly judging the manipulated musical relations (e.g., sensory consonance judgments, timbre or phoneme identification).
}

localization of MEG data, they have provided first indications for the role of inferior frontal cortex in musical structure processing. Unexpected chords containing out-of-key tones evoke an early right anterior-temporal negativity (RATN, Patel et al., 1998 ) or an early right anterior negativity (ERAN) (Koelsch et al., 2000), depending on the construction of the musical sequences. Patel et al. (1998) suggested links between RATN and right frontotemporal circuits reported for working memory of musical material (Zatorre et al., 1994). Both negativities have been compared to a left-lateralized early negativity observed for syntactic incongruities in speech, which is localized in inferior frontal areas within and around Broca's area (Friederici, 2002; Friederici et al., 2000). The involvement of inferior frontal areas in musical structure processing has received further support by MEG (Maess et al., 2001) and fMRI (Koelsch et al., 2002, 2005; Tillmann et al., 2003). An unexpected chord structure containing out-of-key tones evoked bilaterally a magnetic equivalent of the ERAN (magnetic ERAN, mERAN), with its source localized in Broca's area and its right hemisphere homologue (Maess et al., 2001). Using fMRI, Koelsch et al. (2002) confirmed inferior frontal activation for several types of expectancy violations: strongly expected in-key chords were compared to dissonant tone clusters (out-of-key tones without regular chord interval-structure), modulating chords installing a new key (and containing out-of-key tones) and chords played by deviant timbres. Using the musical priming paradigm, Tillmann et al. (2003) reported stronger activation of bilateral inferior frontal areas for unrelated, out-of-key targets than for related targets.

Even if behavioral studies have shown that listeners process relatively subtle differences in musical structures, neurophysiological studies have used somewhat strong tonal manipulations. Related, expected events belonged to the context key, while unexpected events were out-of-key chords (Patel et al., 1998; Tillmann et al., 2003) or contained out-of-key tones (Koelsch et al., 2002, 2005; Maess et al., 2001). This manipulation violates not only the structural regularities of the tonal system, but also implies that solely tones of expected events occur in the context, but not those of unexpected events. This latter feature gives rise to two sensory explanations of the observed context effects in addition to the favored interpretation referring to listener's knowledge-based expectations and musical-syntax processing. First, the difference in activation patterns between expected and unexpected events might be explained by repetition priming. Reduced neural activity for repeated items has been reported for words and pictures (Bergerbest et al., 2004; Buckner et al., 1998; Maccotta and 
Buckner, 2004; Wagner et al., 2000). Expected musical targets might be associated with reduced neural activity since their component tones have already occurred in the context. Second, out-of-key tones do not only create an expectancy violation based on listeners' tonal knowledge, but create sensory dissonance with the other context tones as well (Terhardt, 1984). These perceptual changes define unexpected events as sensory deviants. Since inferior frontal areas are implied in target detection and novelty processing (Kiehl et al., 2001; Linden et al., 1999), the question arises for the music perception data if reported activations reflect the processing of musical deviance (without reference to musical syntax). This hypothesis seems to be supported by the observation that inferior frontal areas are also activated by dissonant chords (Koelsch et al., 2002; Tillmann et al., 2003) and instrumental deviants (Koelsch et al., 2002).

In summary, previous neurophysiological studies do not allow us to distinguish whether inferior frontal areas are sensitive to violations of musical structures or sensitive to violations of sensory features. The main goal of our study was to investigate the implication of inferior frontal areas in musical structure processing by controlling for these alternative interpretations. The musical priming paradigm was used with relatively subtle tonal manipulations: related and less-related targets belonged to the context key and did not create any contextual dissonance (Bigand et al., 2001, 2003; Tillmann et al., 2006). The material allowed us to focus on cognitive priming: targets never occurred in the prime context, and frequency of occurrence of target tones in the prime context even showed a sensory advantage of less-related over related targets. If inferior frontal areas are sensitive to musical structure processing based on listeners' tonal knowledge, stronger activation was predicted for less-related targets, which violate musical syntaxbased expectations. If previously reported activation differences are mainly due to repetition priming or sensory deviance effects, no differences between related and less-related targets should be observed (or even reduced activation for less-related targets). The material consisted of eight-chord sequences with the last chord being the target chord (related or less-related). The material was played with musical instrument samples (creating musical timbres; Tillmann et al., 2006) or sung with artificial syllable sequences (Bigand et al., 2001), and participants performed speeded timbre identification (is the target played by a Timbre A or Timbre B) or phoneme identification (is the target sung on the phoneme /di/ or $/ \mathrm{du} /$ ). For both materials, behavioral data have shown facilitated processing of related over less-related targets in timbre and phoneme identification tasks (Tillmann et al., 2006; Bigand et al., 2001). Using the same experimental materials and methods, the present fMRI study analyzed the neural correlates of cognitive priming that had been investigated in these behavioral studies. Our study made the exploratory investigation whether or not the neural correlates of musical structure processing are modified by the material carrying the musical information (i.e., timbres vs. sung phonemes). Based on previous research investigating musical structure processing (Patel et al., 1998; Koelsch et al., 2002, 2005; Maess et al., 2001; Tillmann et al., 2003), the inferior frontal areas were the focus of interest and a region of interest analysis also investigated an eventual influence of the type of material carrying the musical structure. Beyond inferior frontal areas, previous work led us to expect activation in a set of brain regions, most specifically in anterior and posterior temporal areas (Patel et al., 1998; Koelsch et al., 2002, 2005) and inferior parietal cortex (Koelsch et al., 2005; Tillmann et al., 2003), and to a lesser degree (and depending on susceptibility artifacts of the fMRI signal) also in orbitofrontal cortex (Koelsch et al., 2005). ${ }^{2}$

\section{Methods \\ Participants}

Twenty-one right-handed adults from the participant pool of the Max-Planck-Institute participated after having given written informed consent. One participant was excluded from the analyses because of extreme movements due to neck ache. For the remaining participants (11 females, mean age 25.1 , range $20-$ 33 ), number of years of musical training, as measured by years of instrumental instruction, ranged from 0 to 15 , with a mean of 4.5 $(\mathrm{SD}=5.3)$ and a median of 2.5. Eight of these participants (3 females) had not received any musical instruction (0 years) and were analyzed as a subset of nonmusicians in supplementary analyses.

\section{Materials}

\section{Musical material}

The experimental sequences of Bigand et al. $(2001,2003)$ were used: ${ }^{3}$ Twelve 6-chord sequences (covering the 12 major keys) were completed with two chord pairs to create 8 -chord sequences. These endings were defined in such a way that the last chord (the target) functioned either as a tonic (related condition) or a subdominant chord (less-related condition). A given chord pair defined once the related ending (e.g., $\mathrm{G}-\mathrm{C}$ after a $\mathrm{C}$ Major context) and once the less-related ending (e.g., $\mathrm{G}-\mathrm{C}$ after a $\mathrm{F}$ Major context). The target chord never occurred in the prime context. The number of pitch classes shared with the prime chords was higher for less-related targets (14.75) than related targets (12.5). In all sequences, each of the seven prime chords sounded for $625 \mathrm{~ms}$ and the target (the eighth chord) for $850 \mathrm{~ms}$. For the sung phoneme condition, musical sequences were played with sample voice sounds of the Vocal-Writer software 1.0 (http:// www.kaelabs.com). The 4 notes of a chord were sung by different voices, but on the same syllables. On the basis of 5 consonants and 5 vowels, $22 \mathrm{CV}$-syllables were constructed and assigned in various combinations to the prime chords. One of the prime contexts contained the following syllables: /da fei ku fo fa to kei/. The syllables /di/ and /du/ were always assigned to the target. For the musical timbre condition, the sequences were played by different timbres. To imitate complexity and variety of changing sounds in the phoneme sequences, the prime chords were played by associations of two timbres: the first timbre defined the attack of the sound and the second timbre the sustained portion of the

\footnotetext{
${ }^{2}$ Some of the previously reported activation were expected not to be observed, notably activation in middle frontal gyrus (MFG): In Tillmann et al. (2003), this activation had been attributed to increased monitoring demands for unrelated consonant targets because of the conflict between the dissonance created by the unrelated context and the requested sensory consonance judgments. In the present study, MFG activation should not be replicated with the more subtle context manipulations and experimental tasks on two different dimensions (i.e., timbre and phoneme identification), which did not create a response conflict.

3 Example sequences are available at: http://olfac.univ-lyon1.fr/ bt-sound.html.
} 
sound (e.g., the attack of an acoustic piano was followed by the sustained part of a trumpet sound). Ten timbres were used: organ, guitar, piano, koto (i.e., a Japanese chord instrument) and harpsichord for the attack, and trumpet, oboe, muted trumpet, clarinet and flute for the sustained portion. As for the phoneme sequences, the sound combinations were attributed differently to the prime chords. One of the prime contexts contained the following timbre combinations: organ/trumpet, guitar/oboe, piano/ muted trumpet, koto/trumpet, piano/clarinet, koto/flute, guitar/ oboe. For the timbre identification task, the target was played by either an electric piano sound (Timbre A) or a harpsichord sound (Timbre B). The sound samples were chosen from Miroslav Vitous Symphonic Orchestra samples (monophonic instruments) and Wizoo (polyphonic instruments). The sequences were created with the software Cubase 5.1 (Steinberg) and a Halion sampler (Steinberg). The present article focuses on neural correlates of musical priming in sung and instrumental sequences. As a secondary goal, our study aimed to investigate neural correlates of the processing of sung music (combining musical structures and syllables) and added a third condition with spoken syllables (data not reported here). For the spoken phoneme condition, the strings of syllables of the sung phoneme condition were spoken at the same tempo by synthetic voices of the software MacTTS (Devallois) and the library Mbrola (Dutoit et al., 1996). Four voices (two male, two female) were superposed to produce a sound complexity comparable to the sung sequences.

The material was recorded mono in CD quality $(44.1 \mathrm{kHz}, 16$ bits) with SoundEdit 16 software (Macromedia). The root-mean square power of all sequences was equalized. In the training phase, the test was run with Psyscope 1.2.5 software (Cohen et al., 1993). In the scanning session, the software Presentation 0.8 (Neurobehavioral Systems) was used for stimulus presentation, recording of responses and response time as well as the synchronization with the scanner. Stimuli were presented via the Commander XG soundsystem (Resonance Technology, Inc.), and responses were collected via house-made response boxes.

\section{Procedures}

\section{Training phase}

Participants were trained for the experimental tasks outside the scanner in the following order: spoken phonemes, sung phonemes and musical timbres. They were required to make judgments as quickly and accurately as possible by pressing one of two keys of the computer keyboard. For the task on spoken and sung phonemes, they were trained to identify $/ \mathrm{du} /$ and $/ \mathrm{di} /$ with 4 isolated targets, then with 2 sequences. For the timbre task, they were trained to identify Timbre A and Timbre B with 48 isolated targets, then 4 sequences (the training phase could be repeated if necessary). Accuracy in the timbre task was at $97 \%$ (ranging from $94 \%$ to $100 \%$ ). In the three training parts, an incorrect response was accompanied by an alerting feedback signal and the next trial began when participants pressed a third key. The phoneme $/ \mathrm{du} /$ and the timbre A were associated to the left key (pressed with the left index), and the phoneme / di/ and the timbre B with the right key (pressed with the right index). At the end of the training phase, time line, cues and instructions for the experimental phase in the scanner were presented.

\section{Behavioral procedure in the scanner}

The sequences were presented in short blocks separating tasks (Fig. 2). Each block contained 4 related and 4 less-related sequences with half of the targets being played by Timbre A, half by Timbre $\mathrm{B}$ in the timbre identification task and half of the targets being / $\mathrm{di} /$ and half being / du/ in the phoneme identification task. Sequences were presented in pseudo-random order excluding immediate repetition of 4 same relatedness or response types. The inter-trial interval between sequences was set to 2500 $\mathrm{ms}$ in average with a jitter of $1000 \mathrm{~ms}$. Before the beginning of a short block, the two response types were presented to indicate the upcoming task and to remind participants of the response options (i.e., cues for targets $C T$ ). The first sequence of the block started $5 \mathrm{~s}$ after $C T$. The short blocks were presented in ten groups of three (i.e., sung phonemes, spoken phonemes, musical timbres). Inside a group, a short bloc was followed by $5 \mathrm{~s}$ of silence to indicate participants the end of a task. The order of presentation of material type changed between groups and over participants. Each group was announced by a verbal cue (i.e., cues for group $C G$ ) indicating its position (i.e., first, second ... tenth) to allow participants estimating the time line of the experiment. The end of a group was indicated by a white noise of $250 \mathrm{~ms}$ and was followed $5 \mathrm{~s}$ later by a resting period of $35 \mathrm{~s}$. For spoken, sung and timbre conditions, respectively, 80 sequences were presented (i.e., a total of 240 sequences). Participants were required to judge the phoneme or the timbre of the target as quickly and accurately as possible by pressing one of two buttons of the response box. No feedback was given. The overall duration was $43 \mathrm{~min}$.

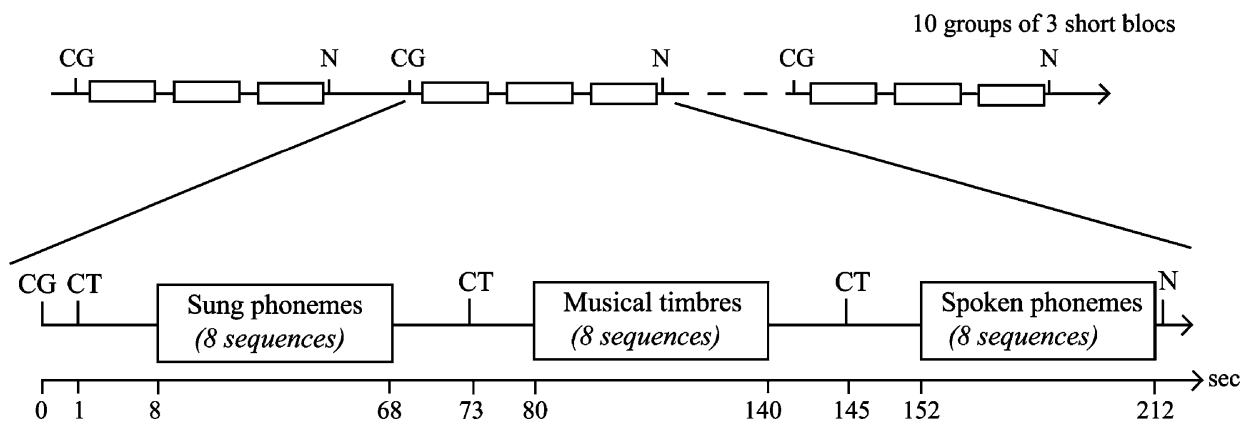

Fig. 2. Schematic presentation of the time course of the experimental session: 10 groups of 3 short blocks (containing 8 sequences of either timbre sequences, sung or spoken phoneme sequences) were separated by rest periods (bordered by $N$ and $C G$ ). $C G$ (Cue Group) is the cue indicating the group's position (i.e., first, second. . ). CT (Cue Targets) indicates the presentation of the two target types: Timbre A and Timbre B for the timbre identification task, di and du (sung or spoken) for the phoneme identification task. $N$ represents the noise bursts indicating the end of the third short block and the beginning of the rest period. The bottom part of the figure displays the construction of one group with approximate timing (for detailed timing information, see Methods). 


\section{Data acquisition}

Data were acquired at 3T on a Siemens' TRIO MRI scanner. During the functional scans (acquired continuously), the BOLD response was measured using a single-shot gradient-echo-planarimaging EPI sequence $(T R=2000 \mathrm{~ms} ; T E=30 \mathrm{~ms}$; flip angle $90^{\circ}$; matrix $64 \times 64$; field of view $192 \times 192 \mathrm{~mm}$ ). Each volume was comprised of twenty-four axial slices with a resolution of $3 \times 3 \times 4 \mathrm{~mm}$ and a spacing of $1 \mathrm{~mm}$. The acquired volume provided whole-brain coverage, except for the bottom part of the cerebellum. Slices were positioned parallel to the bicommissural plane (AC-PC). Prior to the functional scans, an anatomical T1-weighted 2D-image (resolution: $0.8 \times$ $0.8 \times 4.0 \mathrm{~mm}, T R=1300 \mathrm{~ms} ; T E=7.4 \mathrm{~ms}$; flip angle $90^{\circ}$; matrix $256 \times 256$; FOV $192 \times 192 \mathrm{~mm}$ ) and a T1-weighted EPI volume were acquired, both with 24 contiguous slices in the same planes as the functional images. In previous sessions, high-resolution whole-brain anatomical T1-weighted 3D-images had been acquired for each participant.

\section{Data processing and analyses}

Data processing and analyses were conducted using the software package LIPSIA (Lohmann et al., 2001), except for motion correction of functional images, which was realized by Siemens' software prior to image construction. The first 5 EPI volumes were discarded prior to data processing. To correct for the temporal offset between slices acquired in one scan, a cubicspline-interpolation was applied. A temporal high-pass filter with a cutoff frequency of $1 / 216 \mathrm{~Hz}$ was used for baseline correction and a spatial Gaussian filter with $7 \mathrm{~mm}$ FWHM was applied. The anatomical slices were coregistered with the 3-D anatomical images standardized to the Talairach stereotactic coordinate system and transformed by linear scaling and subsequent nonlinear scaling (Thirion, 1998). The transformation parameters obtained were then applied to the functional slices. Slice gap values were interpolated using a trilinear interpolation, generating output data with a spatial resolution of $3 \times 3 \times 3$ $\mathrm{mm}$. Functional data were analyzed for task-related activation using the general linear model (Friston et al., 1995) as implemented in LIPSIA. In the design matrix, regression coefficients were estimated for the five target types time-locked to the beginning of the target (related and less-related targets for sung and timbre sequences, spoken targets), for the three context sequences (the first seven events in sung, timbre and spoken sequences, respectively), for noise bursts, cues and resting periods. The regressors for targets, noise bursts and cues were modeled as events with the canonical haemodynamic response function (Friston et al., 1998). The regressors for contexts and resting periods were modeled as epochs with a boxcar waveform convolved with the same haemodynamic response function. The model equation, including data, design matrix and error term, was convolved with a Gaussian kernel of dispersion of 4s FWHM (Worsley and Friston, 1995). The following contrast images (i.e., estimates of raw-score differences between specified conditions) were generated for each participant: Related targets versus Less-related targets; Sung targets versus Timbre targets; and the interaction between Relatedness (Related/Less-related) and Material (Sung/Timbre). Contrast images of each participant were entered into random effects group analyses using one-sample $t$ tests (Holmes and
Friston, 1998). Resulting $t$ values were transformed into $Z$ scores, and regions with $z>3.09(P<0.001$; uncorrected) and with a volume greater than $270 \mathrm{~mm}^{3}$ (>10 voxels) were reported in Table 1 . Group data were projected on the averaged T1-weighted anatomical image (based on the T1 3D-images of all participants) and activated areas were identified with the atlas of Talairach and Tournoux (1988) and the atlas of Duvernoy (1999). For the Region of Interest time course analyses, trial averages of the BOLD response time course were computed by time shifting all trials of related and lessrelated targets (for sung phonemes and musical timbres, respectively) such that the onset times were 0 . For all contrasts, supplementary second-level analyses based on Bayesian statistics were performed (Neumann and Lohmann, 2003). Bayesian approaches provide an alternative to classical analyses based on statistical significance tests. Instead of testing the estimated probability to obtain this contrast activation with the null hypothesis of no activation being true (i.e., $P$ values), the Bayesian approach directly infers the probability that a contrast between two experimental conditions is larger than zero. When applied to second-level analyses, the posterior probability of the group's contrast to be larger than zero is calculated based on the parameter estimations for the individual participants on the first level (i.e., on the beta-values of the general linear model). The resulting posterior probability maps then provide a measure of the reliability of activation differences in the group (expressed as a percentage value between 0 and 100). Bayesian inferences are not susceptible to problems of multiple comparisons thanks to direct computation of the probabilities. Bayesian statistics considers only voxels for which signal was measured in all participants.

Since the participant pool included participants with varying levels of musical expertise, additional analyses focusing on nonmusicians were performed for behavioral data and fMRI data (i.e., for the contrast Related targets versus Less-related targets and associated ROI analyses). These analyses investigated that data patterns were also observed for nonmusician listeners with implicitly acquired musical knowledge and were not restricted to explicitly trained musician listeners.

\section{Results}

\section{Behavioral data}

Accuracy was high overall, but slightly higher for sung phonemes ( $94 \%$ for related and less-related targets) than for musical timbres $(88 \%$ for related and $87 \%$ for less-related targets). Data of one subject were excluded from these percentages, as one third of the responses (including spoken targets) were not recorded. For sung and timbre targets, correct response times (Fig. 3) were analyzed by a $2 \times 2$ ANOVA with Relatedness (Related/Less-Related) and Material (Sung Phonemes/Musical timbres) as within-subjects factors. Response times were faster for related targets than for less-related targets, $F(1,19)=32.65, P<0.001$. This difference was more pronounced for musical timbres, $F(1,19)=4.51, P<0.05$, but was significant for both timbres, $F(1,19)=28.88, P<$ 0.001 and sung phonemes, $F(1,19)=12.32, P<0.01$. Overall, response times were faster for sung phonemes than for timbres, $F(1,19)=49.35, P<0.0001$. An additional analysis focusing 
Table 1

Talairach coordinates of significant activations for comparisons linked to the musical targets $\left(P<0.001\right.$, uncorrected with peak $Z$ scores and mm ${ }^{3}$-extent): effect of relatedness (Related vs. Less-Related) of material (Timbre vs. Sung) and their interaction

\begin{tabular}{|c|c|c|c|c|c|c|c|c|c|c|}
\hline Contrast & Lobe & Region & $\mathrm{BA}$ & Hemisphere & $x$ & $y$ & $z$ & $Z$ score & $\mathrm{mm}^{3}$ & Bayes \% \\
\hline \multicolumn{11}{|c|}{ Related-Less-Related: no significant activation } \\
\hline \multirow[t]{12}{*}{ Less-Related-Related: } & \multirow[t]{6}{*}{ Frontal } & IFG/pars opercularis & \multirow[t]{4}{*}{$44 / 45$} & Right & 43 & 15 & 3 & 3.80 & \multirow[t]{2}{*}{$1377^{\mathrm{a}}$} & 99.93 \\
\hline & & Insula (anterior) & & Right & 40 & 12 & -3 & 3.84 & & 99.87 \\
\hline & & IFS/white matter & & Right & 28 & 36 & 18 & 3.71 & \multirow[t]{3}{*}{$1350^{\mathrm{a}}$} & 99.98 \\
\hline & & SFS & & Right & 31 & 36 & 30 & 3.65 & & 99.74 \\
\hline & & Anterior cingulate & \multirow[t]{2}{*}{32} & Right & 13 & 36 & 24 & 3.56 & & 99.95 \\
\hline & & Posterior orbital gyrus & & Right & 37 & 12 & -15 & 4.02 & 405 & $\begin{array}{l}97.95 / 99.39 \\
\text { (see Results) }\end{array}$ \\
\hline & \multirow[t]{4}{*}{ Temporal } & $\begin{array}{l}\text { STG anterior/planum } \\
\text { polare }\end{array}$ & $22 / 38$ & Left & -47 & 6 & -6 & 4.14 & 297 & 99.91 \\
\hline & & STS & & Left & -47 & -33 & 9 & 4.22 & $1215^{\mathrm{a}}$ & 99.99 \\
\hline & & STG & & Left & -56 & -30 & 12 & 3.58 & & 99.51 \\
\hline & & & 37 & Left & -44 & -57 & 9 & 3.76 & 729 & 99.99 \\
\hline & \multirow[t]{2}{*}{ Parietal } & \multirow[t]{2}{*}{$\begin{array}{l}\text { Supramarginal } \\
\text { gyrus }\end{array}$} & \multirow[t]{2}{*}{40} & \multirow[t]{2}{*}{ Right } & 61 & -30 & 36 & 4.32 & \multirow[t]{2}{*}{$1701^{\mathrm{a}}$} & $\begin{array}{l}96.30 \\
\left(\begin{array}{lll}60 & -30 & 35\end{array}\right)\end{array}$ \\
\hline & & & & & 49 & -33 & 36 & 3.78 & & 99.98 \\
\hline \multicolumn{11}{|l|}{ Timbre-Sung: no significant activation } \\
\hline \multirow[t]{4}{*}{ Sung-Timbre: } & \multirow[t]{3}{*}{ Frontal } & SFG medial & \multirow[t]{2}{*}{10} & Right & 4 & 54 & 18 & 4.24 & 378 & 99.73 \\
\hline & & $\begin{array}{l}\text { SFG medial / } \\
\text { white matter }\end{array}$ & & Right & 16 & 57 & 9 & 4.07 & 432 & $\begin{array}{l}99.99 \\
(165410)\end{array}$ \\
\hline & & SFG medial & 10 & Left & -8 & 57 & 12 & 3.66 & 405 & $\begin{array}{l}99.51 \\
\left(\begin{array}{lll}-9 & 57 & 12\end{array}\right)\end{array}$ \\
\hline & \multirow[t]{2}{*}{ Parietal } & Inferior Parietal Gyrus & \multirow[t]{2}{*}{40} & Left & -56 & -48 & 42 & 3.56 & 378 & $\begin{array}{l}99.88 \\
(-53-4742)\end{array}$ \\
\hline Interaction Relatedness $\times$ Material & & $\begin{array}{l}\text { Post. parahippocampal } \\
\text { gyrus/ lingual gyrus }\end{array}$ & & Right & 25 & -57 & 3 & 4.09 & 648 & 99.90 \\
\hline
\end{tabular}

The last column indicates posterior probabilities from Bayesian statistics estimating the reliability of activation differences (expressed as a percentage value between 0 and 100).

${ }^{a}$ Denotes activation clusters that are specified by additional coordinates indicated in the following line(s). For probabilities, coordinates differing from peak coordinates are indicated in brackets (see Results for details).

on the subset of nonmusicians confirmed a main effect of Relatedness, $F(1,7)=15.54, P<0.01$ and of Material, $F(1,7)=$ 17.94, $P<0.01$.

\section{fMRI data}

\section{Musical relatedness}

Contrasts opposing related and less-related targets revealed significantly stronger activation only for less-related targets. This activation included frontal, insular, temporal and parietal regions (Fig. 4). Of particular interest was the activation of right inferior frontal gyrus (deep frontal operculum) and anterior insula. In the left hemisphere, a local maximum in the analogous region $(-41186, z=3.15)$ did not exceed extent threshold, but was part of a significant activation cluster at reduced significance threshold $(P<0.005)$. In the right hemisphere, frontal activation foci were also observed along the inferior and superior frontal sulci (IFS, SFS) and the lateral posterior orbital gyrus. In the temporal lobe, significant BOLD signal increases were observed in the left planum polare, STS/ STG and posterior MTG. In the parietal lobe, activation was significant in the right supramarginal gyrus SMG.

For the right fronto-insular activation, the relatedness effect was further investigated with Region of Interest analyses separating timbre targets and sung targets. Increased activation was observed for less-related targets independently of the material (Fig. 5). A supplementary analysis of statistical maps including only the subset of nonmusicians confirmed a local maximum in right frontal operculum (40 $126, z=3.27)$ with an extent of $108 \mathrm{~mm}^{3}$. At reduced threshold of $P<0.005$, this activation maximum was included in a larger activation area comprising anterior insula $\left(3051 \mathrm{~mm}^{3}\right)$.

\section{Materials}

Contrasts investigating the effect of material (sung vs. timbre targets) revealed significant activation only for sung targets minus timbre targets. BOLD signal increases were observed for sung targets in the medial superior frontal gyrus (bilaterally) and the left inferior parietal gyrus.

\section{Interaction relatedness $\times$ material}

The contrast investigating whether the relatedness effect differed between sung and timbre targets revealed only a single activation focus in right posterior parahippocampal gyrus/lingual gyrus.

The probabilities based on Bayesian statistics indicating the reliability of differences are listed for peak coordinates of all clusters in Table 1. For some very lateral coordinates that did not have signal for all participants, probabilities were indicated for neighboring coordinates. For the orbito-frontal cluster, this procedure was not possible since one participant showed a susceptibility artifact in this region. An additional analysis excluding this participant showed a probability of $97.95 \%$ and $99.39 \%$ at $3712-14$ and $3612-14$, respectively. For all 


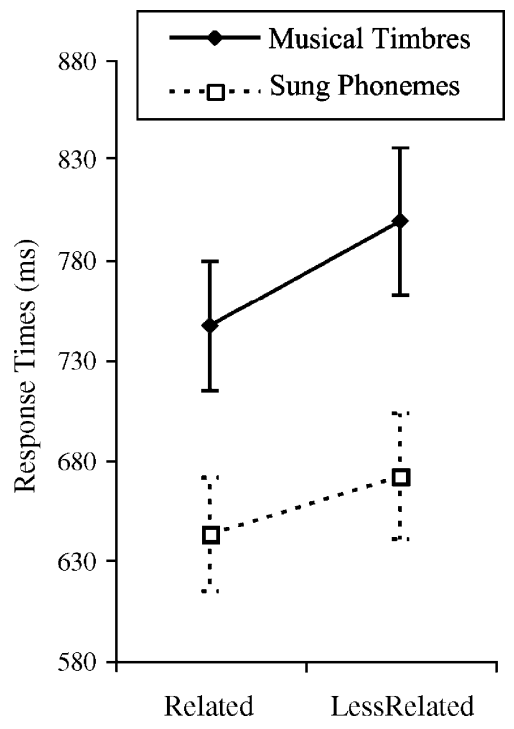

Fig. 3. Correct response times for targets played by musical timbres or sung phonemes as a function of their musical relatedness (related/less related) and averaged over the sequence set and participants. Error bars indicate standard errors over participants.

other areas, clusters of strongest probabilities in the posterior probability maps (based on all participants) were localized in areas corresponding to the activation clusters observed in the z-maps.

\section{Discussion}

Neurophysiological research investigating musical syntax processing has shown that musically unexpected events increased activation of inferior frontal areas in comparison to expected ones (Koelsch et al., 2002, 2005; Maess et al., 2001; Tillmann et al., 2003). However, the strong musical manipulations left space for alternative explanations in terms of repetition priming or sensory

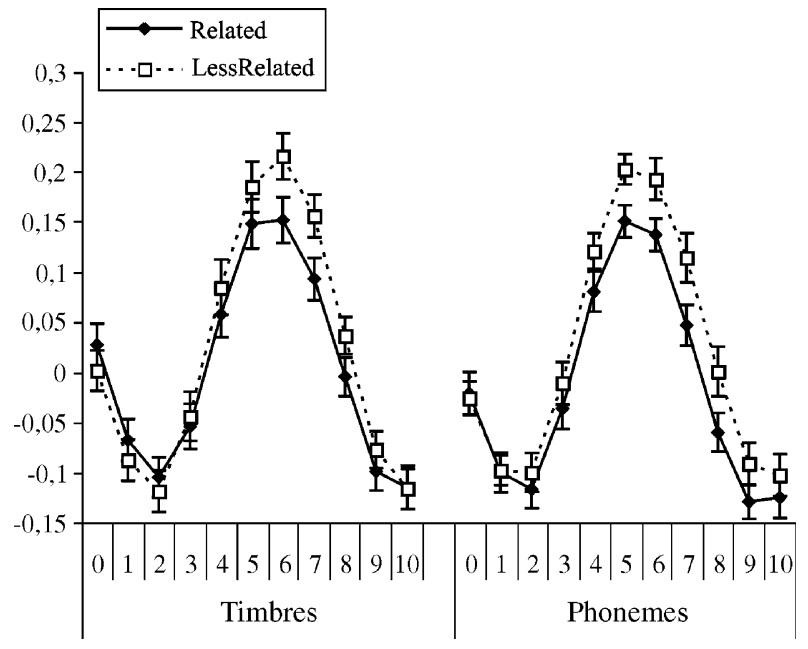

Fig. 5. The time course of the BOLD response (percent signal change) for related and less-related targets played by musical timbres (left) and sung phonemes (right) in the right inferior frontal activation (frontal operculum and adjacent anterior insula, see Table 1), with 0 representing the onset of the target chord (in steps of 1s). Error bars indicate standard errors over participants.

deviance detection. Our study investigated the implication of inferior frontal areas in musical syntax processing by focusing on knowledge-based expectations (as in previous behavioral studies, Bigand et al., 2001, 2003): expected and less-expected target events were in-key chords that differed in their musical relatedness to the prime context, and the targets' frequency of occurrence in the prime sequences was controlled. The behavioral data acquired in the scanner replicated facilitated processing of related targets over less-related targets in both phoneme and musical timbre identification tasks (Bigand et al., 2001; Tillmann et al., 2006). The fMRI data showed an activation pattern that is overall comparable to activations reported for stronger musical expectancy violations (Koelsch et al., 2002, 2005; Tillmann et al., 2003). In addition to inferior frontal and anterior insula activation, the processing of

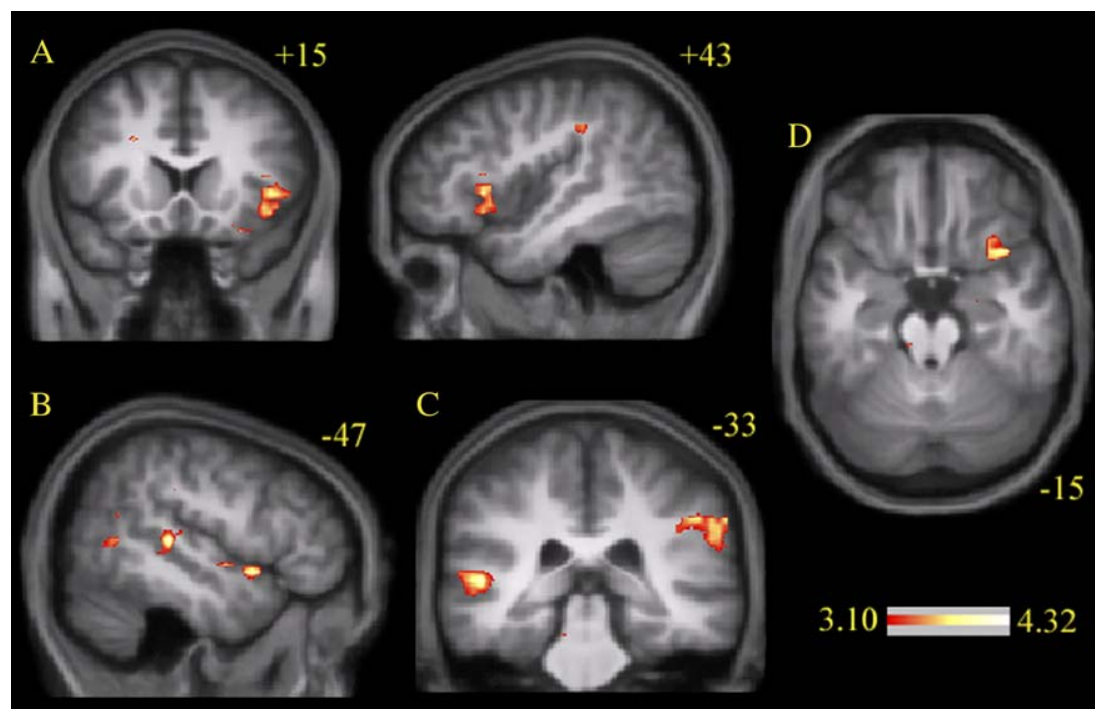

Fig. 4. Group image showing significant differences in BOLD signal $(P<0.001 ; z>3.09)$ of less-related over related targets. (A) inferior frontal activation (frontal operculum, anterior insula), (B) activation in left anterior STG, STS and posterior MTG, (C) activation in left STS and right SMG and (D) right orbital gyrus activation. Activations are superimposed on the averaged T1-weighted anatomical images of all participants. 
less-related targets evoked stronger activation in additional frontal areas as well as temporal and parietal areas.

\section{Inferior frontal cortex}

In comparison to related targets, less-related targets increased activation in inferior frontal cortex (i.e., inferior frontal gyrus, frontal operculum extending into the anterior insula). Activations were mainly observed in the right hemisphere, but with reduced statistical threshold also in the left hemisphere. This finding is in agreement with previously reported inferior frontal activation for musically unexpected events, with a right-hemispheric asymmetry (Maess et al., 2001; Koelsch et al., 2002, 2005; Tillmann et al., 2003). We suggest that the same circuitry is involved, but less strongly activated due to the more subtle musical violations, which were purely syntactic and excluded sensory violations. For musical targets, the neural correlates of expectancy violations were observed independently of whether musical structures were carried by timbres or sung syllables. For both materials, listeners form musical expectations, which slow down the processing of lessrelated events and increase activation in inferior frontal areas.

The controlled construction of the material allowed us to focus on neural correlates of cognitive, knowledge-driven processes in musical priming and to discard the evoked alternative, sensorydriven explanations that are based on deviance processing (Kiehl et al., 2001; Linden et al., 1999) and repetition suppression (e.g., Maccotta and Buckner, 2004). Less-related targets did not define a sensory deviant or auditory oddball, and neither less-related nor related targets occurred in the context. Moreover, if repetition suppression was determining the data pattern, activation should be reduced for less-related targets because component tones of lessrelated targets occurred slightly more often in the prime context than those of related targets. Our outcome thus suggests that previously reported inferior frontal activations were caused neither solely by deviance processing of unrelated events nor by repetition suppression (i.e., sensory priming) due to related events (Tillmann et al., 2003; Koelsch et al., 2002, 2005). The musical violations used here showed that increased neural activity for unrelated and less-related targets appear to be due to cognitive, knowledge-driven processes. Listeners' knowledge on prototypical musical structures allows musical expectancy formation and influences perception of incoming musical events: processing unusual, unexpected structures requires more neural resources than processing more familiar and prototypical structures.

Our outcome suggests that the frontal operculum is sensitive to the processing of music-syntactic relations. Right frontal involvement has been observed for the processing of musical relations in melodies (Krumhans1, 2003; Platel et al., 1997; Zatorre et al., 1994), mental imagery for songs (Halpern and Zatorre, 1999), singing (Jeffries et al., 2003) as well as rhythm and pitch discrimination tasks (Rao et al., 2001). Our finding can be integrated to other data showing that inferior fronto-lateral areas participate in nonlinguistic processes (Adams and Janata, 2002; Griffiths et al., 1999; Müller et al., 2001; Pugh et al., 1996) apart from their roles in semantic (Poldrack et al., 1999; Wagner et al., 2000), syntactic (Embick et al., 2000; Fiebach et al., 2005; Meyer et al., 2000; Petersson et al., 2004) and phonological functions (Poldrack et al., 1999; Pugh et al., 1996). In the musical priming situation, frontal opercular activation to the same acoustic event (i.e., target) changes with the event's musical function. These data suggest that the fronto-opercular cortex is not only processing acoustic features (timbre, phonemes), but is sensitive to the musical context, notably to the target's musical relatedness and listener's contextual expectations. For word processing, increased activation in left pars opercularis has been similarly shown after expectancy violations in incongruent (versus congruent) sentences (Cardillo et al., 2004). The use of artificial grammars made out of syllable sequences allowed specifying that violations of local structures increased activation in the left frontal operculum and violations of hierarchical structures additionally increased activation in left BA44/45 (Friederici et al., 2006). In our present study, violations of musical expectations increased activation in parts of both areas in the right hemisphere. Future research has to further specify functional subregions of right inferior frontal cortex in response to musical or, more generally, nonverbal auditory structure processing.

Frontal operculum and ventrolateral prefrontal cortex have been observed in processing temporal structures and predicting future sequential events (Coull, 2004; Fuster, 2001; Janata and Grafton, 2003; Rao et al., 2001; Schubotz et al., 2000). For example, increased activation was reported in fronto-opercular cortex when participants indicated violations of previously encountered rhythmic patterns (Schubotz and von Cramon, 2001a) and specific event successions (Schubotz and von Cramon, 2001b). In these studies, predictions were based on systematic regularities or repeated patterns learned in the experimental session. In our study, predictions are based on listeners' long-term knowledge (i.e., existing before the experiment). The context activates listeners' musical knowledge that allows generating expectations for future events and their temporal moment of occurrence (e.g., Jones and Boltz, 1989). The expectations are stronger for related targets (i.e., tonic chord) than less-related targets because the tonic is functionally at the top of the tonal hierarchy and provides an adequate ending of the sequence (i.e., giving the impression of closure). Violations of these expectations evoke increased activation in inferior frontal areas. Data for musical, linguistic, phonological and temporal processing suggest a role of inferior frontal areas in detection and recognition of structural regularities as well as for the integration of sequential events over time. Future research has to specify whether their functional relevance depends on the activation status of other connected brain regions (i.e., integration in an overall network) or on specialized regions in these relatively large areas (i.e., with some being specialized and others being domain-general). ${ }^{4}$

\section{Anterior insula}

As in previous studies investigating neural correlates consecutive to, albeit stronger, musical expectancy violations (Koelsch et al., 2002; Tillmann et al., 2003), the observed fronto-opercular activation extended into the anterior insula. Connections have been described between the insula and the frontal operculum (Ardila,

\footnotetext{
${ }^{4}$ The inferior frontal activation for musical syntax processing can be integrated in a declarative/procedural model, initially developed for language, but open for domain-generality (Ullmann, 2001). This model distinguishes a mental lexicon (i.e., declarative memory system) from a computational "mental grammar" (i.e., procedural memory system). The procedural memory (with neural correlates including frontal cortex and basal ganglia) subserves the implicit learning of regularities, rules and abstract representations, including syntax structures.
} 
1999; Mesulam and Mufson, 1993), and also with auditory regions (Liegeois-Chauvel et al., 1999). Bamiou et al. (2003) suggest that the insula defines an additional auditory processing center, which is involved in allocating auditory attention and in sound sequence processing (rhythm, prosody, phonological features). This hypothesis was supported by the observation of increased anterior insula activation for the perception of melodies (Krumhansl, 2003; Platel et al., 1997; Zatorre et al., 1994), moving sound stimuli (Griffiths et al., 1999), environmental sounds (Adams and Janata, 2002; Engelien et al., 1995) and for pitch discrimination (Wong et al., 2004). In our paradigm, participants processed complex auditory sequences and effectuated a perceptual task on the target. The increased anterior insula activation consecutive to less-expected musical events (in contrast to expected ones) might reflect the enhanced attention and sound processing due to the expectancy violation.

\section{Orbitofrontal cortex}

This cortical area is densely connected to limbic areas (Zald and Kim, 1996). Increased activation of posterolateral orbital gyrus has been reported for unpleasant auditory and olfactory stimuli (Frey et al., 2000; Gottfried et al., 2002) and for memory of stimuli with negative valence (Lewis et al., 2005; Markowitsch et al., 2003). For expectancy violations of learned predictive cues, orbitofrontal activation has been previously interpreted as serving as interface between cognition and affect (Nobre et al., 1999). The orbitofrontal activation consecutive to expectancy violations of musical syntax (see also Koelsch et al., 2005) might also play a role for affective experiences in music, ${ }^{5}$ probably together with the insula, which is part of the paralimbic system and is connected to a multitude of limbic structures (Gruber et al., 2002). When participants were listening to musical pieces specifically selected for their strong emotional response, orbitofrontal and insula activation has been reported to correlate with participants' pleasure (i.e., chills) (Blood and Zatorre, 2001).

\section{Temporal cortex}

Processing less-related targets increased activation in left posterior STS (encompassing STG) in comparison to related targets. Stronger musical expectancy violation (i.e., including out-of-key tones) evoked bilateral STS (and STG) activation (Koelsch et al., 2005). STS activation has also been reported for the perception of phonological features in speech (e.g., Binder et al., 1994) and of phonetic cues irrespective of intelligibility (Scott et al., 2000). Wise et al. (2001) argue for a role of left posterior STS in the buffering and representing of incoming sound structures over short time periods, notably for word perception. Data on music perception suggest that this role extends to the perception of other acoustic sequences, notably with enhanced implication for the perception of unexpected structures. Corroborative evidence for the role of posterior superior temporal lobe (STG and STS) in online language perception has been reported for sentence processing, notably with increased activation for sentences with either syntactic or semantic violations (Friederici et al., 2003). These findings suggest that for language and music, unexpected or less-

\footnotetext{
5 The specific link between musical expectations and musical expressiveness and emotion has been established by Meyer (1956).
}

expected items render difficult the processes of on-line integration, requiring enhanced implication of posterior temporal areas.

In addition to the STS activation, less-related targets evoked increased activation of posterior MTG in comparison to related targets (see also Koelsch et al., 2005). Together with data on language perception, this observation suggests that posterior MTG activation might assist in processes linked to the integration of sequential events over time. Increased posterior MTG activation was observed for complex sentence structures with increased parsing difficulty (Constable et al., 2004). The implication of posterior MTG in short-term memory has been observed in discrimination tasks (on consonants, rhymes and tones of Chinese syllables) requesting listeners to compare first and last elements of triplets (Gandour et al., 2003).

\section{Parietal cortex}

The increased right SMG activation for less-related targets can be interpreted as part of a fronto-temporo-parietal network of auditory attention, which has been observed for simple acoustic materials (Benedict et al., 1998; Pugh et al., 1996; Zatorre et al., 1999) and musical materials (Janata et al., 2002; Satoh et al., 2001). In Pugh et al. (1996), inferior parietal activation was observed to a stronger extent in the right hemisphere for both phoneme and pitch judgments. Our outcome corroborates this observation with right-sided activation independently of the task (phoneme and timbre identification). Beyond attention, SMG activation has been reported for working memory of auditory information (Gaab et al., 2003; Paulesu et al., 1993; Zatorre et al., 1994), with sometimes a right-lateralization for nonverbal auditory stimuli (Gaab and Schlaug, 2003; Zatorre et al., 1994). Enhanced participation of short-term memory is required by the processing of unusual, less-related musical structures in comparison to more prototypical structures. The neural basis of these working-memory processes might extend to the activation of the temporal (i.e., STG/ STS) areas, as suggested by the temporal-parietal system proposed in the speech perception model by Hickok and Poeppel (2000, 2004).

\section{Conclusion}

Our study investigated the neural correlates of cognitive priming in musical sequences, which were either played by musical instruments or sung on syllables. The main outcome was the increased activation of inferior frontal cortex (i.e., inferior frontal gyrus, frontal operculum, anterior insula) for musical expectancy violation without sensory violations. The construction of the musical sequences allowed us to interpret the activation patterns in terms of musical syntax processing, which requests listeners' knowledge about the Western tonal system. It thus discards alternative sensory-driven explanations of expectancy violations and their neural correlates, which are based on sensory deviance processing (acoustic oddball) or repetition suppression.

In combination with results of Koelsch et al. (2005), our data pattern further suggests that musical syntax processing includes not only frontal opercular and anterior insula activation, but the integration of these frontal areas in an overall neural system. The activation overlap between the two studies shows a set of brain areas that includes frontolateral and orbitofrontal areas, the anterior insula, temporal (aSTG, posterior STS/STG and MTG) and right 
parietal (SMG) areas. ${ }^{6}$ Further investigation of this neural system and eventual functional connectivity patterns becomes particularly important in light of the recent observation that IR, a patient with right inferior frontal lesions, showed a musical priming effect (Tillmann, Bigand and Peretz, submitted). For language processing (see Friederici, 2002 for an overview), the reported set of brain areas includes IFG (frontal operculum), aSTG, posterior STG/STS and parietal areas (mainly reflected in posterior ERP components). The parallels in the described neural systems suggested for music and language (Besson and Schön, 2003; Koelsch, 2005; Patel et al., 1998) can be interpreted in light of their structural similarities: sequences of musical events and sequences of linguistic events are described as hierarchically structured and based on syntactic-like organizations (Lerdahl and Jackendoff, 1983; Patel, 2003). The overlap in neural correlates might reflect processes necessary for structural integration, notably over time.

\section{Acknowledgments}

We thank Stefan Zysset and Karsten Müller for help with LIPSIA and design preparation, Jane Neumann for help with Bayesian analyses and Daniela Sammler for scheduling participants. This research was supported in part by the program CNRS/ MPG "Cooperation 2003" and the CNRS program "Cognition and Information Processing”.

\section{References}

Adams, R.B., Janata, P., 2002. A comparison of neural circuits underlying auditory and visual object categorization. NeuroImage 16, 361-377.

Ardila, A., 1999. The role of insula in language: an unsettled question. Aphasiology 13, 79-87.

Bamiou, D.-E., Musiek, F.E., Luxon, L.M., 2003. The insula (Island of Reil) and its role in auditory processing. Literature review. Brain Res. Rev. 42, 143-154.

Benedict, R.H.B., Lockwood, A.H., Shucard, J.L., Shucard, D.W., Wack, D., Murphy, B.W., 1998. Functional neuroimaging of attention in the auditory modality. NeuroReport 9, 121-126.

Bergerbest, D., Ghahremani, D.G., Gabrieli, J.D., 2004. Neural correlates of auditory repetition priming: reduced fMRI activation in the auditory cortex. J. Cogn. Neurosci. 16, 966-977.

Besson, M., Macar, F., 1987. An event-related potential analysis of incongruity in music and other non-linguistic contexts. Psychophysiology 24 (1), 14-25.

Besson, M., Schön, D., 2003. Comparison between language and music. In: Peretz, I., Zatorre, R.J. (Eds.), The Cognitive Neuroscience of Music. Oxford Univ. Press, Oxford, p. 269-293.

Besson, M., Faïta, F., Requin, J., 1994. Brain waves associated with musical incongruities differ for musicians and non-musicians. Neurosci. Lett. $168,101-105$

Bharucha, J.J., Stoeckig, K., 1987. Priming of chords: spreading activation or overlapping frequency spectra? Percept. Psychophys. 41 (6), 519-524.

\footnotetext{
${ }^{6}$ The data of the two studies differ in hemispheric weighting, notably frontal and temporal activation were reported bilaterally for strong musical violations (Koelsch et al., 2005), while bilateral activation emerged only at lower statistical thresholds for the finer violations. The difference might also be partly due to the continued acquisition used in the present study versus the clustered acquisition in Koelsch et al. (2005) allowing for stimulus presentations on silent background.
}

Bigand, E., Pineau, M., 1997. Global context effects on musical expectancy. Percept. Psychophys. 59 (7), 1098-1107.

Bigand, E., Madurell, F., Tillmann, B., Pineau, M., 1999. Effect of global structure and temporal organization on chord processing. J. Exp. Psychol. Hum. Percept. Perform. 25 (1), 184-197.

Bigand, E., Tillmann, B., Poulin, B., D’Adamo, D.A., Madurell, F., 2001. The effect of harmonic context on phoneme monitoring in vocal music. Cognition 81 (1), B11-B20.

Bigand, E., Poulin, B., Tillmann, B., D’Adamo, D., 2003. Cognitive versus sensory components in harmonic priming effects. J. Exp. Psychol. Hum. Percept. Perform. 29 (1), 159-171.

Bigand, E., Tillmann, B., Manderlier, D., Poulin, B., 2005. Repetition priming: is music special? Q. J. Exp. Psychol. 58A (8), 1347-1375.

Binder, J.R., Rao, S.M., Hammeke, T.A., Yetkin, F.Z., Jesmanowicz, A., Bandettini, P.A., et al., 1994. Functional magnetic resonance imaging of human auditory cortex. Ann. Neurol. 35, 662-672.

Blood, A.J., Zatorre, R.J., 2001. Intensely pleasurable responses to music correlate with activity in brain regions implicated with reward and emotion. Proc. Natl. Acad. Sci. 98, 11818-11823.

Buckner, R.L., Goodman, J., Burock, M., Rotte, M., Koutstaal, W., Schacter, D., et al., 1998. Functional-anatomic correlates of object priming in humans revealed by rapid presentation event-related fMRI. Neuron 20, 285-296.

Cardillo, E.R., Aydelott, J., Matthews, P.M., Devlin, J.T., 2004. Left inferior prefrontal cortex activity reflects inhibitory rather than facilitatory priming. J. Cogn. Neurosci. 16, 1552-1561.

Cohen, J., MacWhinney, B., Flatt, M., Provost, J., 1993. PsyScope: an interactive graphic system for designing and controlling experiments in the psychology laboratory using Macintosh computers. Behav. Res. Methods Instrum. Comput. 25, 257-271.

Constable, R.T., Pugh, K.R., Berroya, E., Mencl, W.E., Westerveld, M., Ni, W., et al., 2004. Sentence complexity and input modality effects in sentence comprehension: an fMRI study. NeuroImage 22, 11-21.

Coull, J.T., 2004. fMRI studies of temporal attention: allocating attention within, or towards, time. Brain Res. Cogn. Brain Res. 21, 216-226.

Dutoit, T., Pagel, V., Pierret, N., Bataille, F., Van Der Vreken, O., 1996. The MBROLA project: towards a set of high-quality speech synthesizers free of use for non-commercial purposes. Paper presented at the 7th International Conference on Spoken Language Processing, Philadelphia.

Duvernoy, H.M., 1999. The Human Brain: Surface, Three-Dimensional Sectional Anatomy with MRI, and Blood Supply, 2nd ed. SpringerVerlag, Wien.

Embick, E., Marantz, A., Miyashita, Y., O’Neil, W., Sakai, K.L., 2000. A syntactic specialization for Broca's area. Proc. N. Y. Acad. Sci. 97, 6150-6154.

Engelien, A., Silbersweig, D., Stern, E., Huber, W., Doring, W., Frith, C., et al., 1995. The functional anatomy of recovery from auditory agnosia. A PET study of sound categorization in a neurological patient and normal controls. Brain 118, 1395-1409.

Fiebach, C.J., Schlesewsky, M., Lohmann, G., von Cramon, D.Y., Friederici, A.D., 2005. Revisiting the role of Broca's area in sentence processing: syntactic integration versus syntactic working memory Hum. Brain Mapp. 24, 79-91.

Francès, R., 1958. La perception de la musique, 2nd ed. Vrin, Paris.

Frey, S., Kostopoulos, P., Petrides, M., 2000. Orbitofrontal involvement in the processing of unpleasant auditory information. Eur. J. Neurosci. 12, $3709-3712$.

Friederici, A.D., 2002. Towards a neural basis of auditory sentences processing. Trends Cogn. Sci. 6 (2), 78-84.

Friederici, A.D., Meyer, M., von Cramon, D.Y., 2000. Auditory language comprehension: an event-related fMRI study on the processing of syntactic and lexical information. Brain Lang. 74 (2), 289-300.

Friederici, A.D., Rüschemeyer, S.-A., Hahne, A., Fiebach, C.J., 2003. The role of left inferior frontal and superior temporal cortex in sentence comprehension: localizing syntactic and semantic processes. Cereb. Cortex 13, 170-177. 
Friederici, A.D., Bahlmann, J., Heim, S., Schubotz, R.I., Anwander, A., 2006. The brain differentiates human and non-human grammars: functional localization and structural connectivity. Proc. Natl. Acad. Sci. U. S. A. 103 (7), 2458-2463.

Friston, K.J., Holmes, A.P., Worsley, K.J., Poline, J.P., Frith, C.D., Frackowiak, R.S.J., 1995. Statistical parametric maps in functional imaging: a general linear approach. Hum. Brain Mapp. 2, $189-210$.

Friston, K.J., Fletcher, P., Josephs, O., Holmes, A., Rugg, M.D., Turner, R., 1998. Event-related fMRI: characterizing differential responses. NeuroImage 7, 30-40.

Fuster, J.M., 2001. The prefrontal cortex-An update: time is of the essence. Neuron 30, 319-333.

Gaab, N., Schlaug, G., 2003. The effect of musicianship on pitch memory in performance matched groups. NeuroReport 14 (18), 2291-2295.

Gaab, N., Gaser, C., Zaehle, T., Jancke, L., Schlaug, G., 2003. Functional anatomy of pitch memory-An fMRI study with spare temporal sampling. NeuroImage 19, 1417-1426.

Gandour, J., Xu, Y., Wong, D., Dzemidzic, M., Lowe, M., Li, X., 2003. Neural correlates of segmental and tonal information in speech perception. Hum. Brain Mapp. 20, 185-200.

Gottfried, J.A., Deichmann, R., Winston, J.S., Dolan, R.J., 2002. Functional heterogeneity of human olfactory cortex: an event-related functional magnetic resonance imaging study. J. Neurosci. 22, 10819-10828.

Griffiths, T.D., Johnsrude, I., Dean, J.L., Green, G.G.R., 1999. A common neural substrate for the analysis of pitch and duration pattern in segmented sound? NeuroReport 10, 3825-3830.

Gruber, O., Arendt, T., von Cramon, D.Y., 2002. Neurobiologische Grundlagen der Stirnfunktionen. In: Foerstl, H. (Ed.), Frontalhirn: Funktionen und Erkrankungen. Springer, Berlin, p. 21-47.

Halpern, A.R., Zatorre, R.J., 1999. When that tune runs through your head: a PET investigation of auditory imagery for familiar melodies. Cereb. Cortex 9 (7), 697-704.

Hickok, G., Poeppel, D., 2000. Towards a functional neuroanatomy of speech perception. Trends Cogn. Sci. 4 (4), 131-138.

Hickok, G., Poeppel, D., 2004. Dorsal and ventral streams: a framework for understanding aspects of the functional anatomy of language. Cognition 92, 67-99.

Holmes, A.P., Friston, K.J., 1998. Generalisability, random effects and population inference. NeuroImage, vol. 7, p. S754.

Janata, P., 1995. ERP measures assay the degree of expectancy violation of harmonic contexts in music. J. Cogn. Neurosci. 7, 153-164.

Janata, P., Grafton, S.T., 2003. Swinging in the brain: shared neural substrates for behaviors related to sequencing and music. Nat. Neurosci. 6 (7), 682-687.

Janata, P., Tillmann, B., Bharucha, J.J., 2002. Listening to polyphonic music recruits domain-general attention and working memory circuits. Cogn. Affect. Behav. Neurosci. 2, 121-140.

Jeffries, K.J., Fritz, J.B., Braun, A.R., 2003. Words in melody: an $\mathrm{H}_{2}^{15} \mathrm{O}$ PET study of brain activation during singing and speaking. NeuroReport 14, 749-754.

Jones, M.R., Boltz, M., 1989. Dynamic attending and responses to time. Psychol. Rev. 96, 459-491.

Kiehl, K.A., Laurens, K.R., Duty, T.L., Forster, B.B., Liddle, P.F., 2001. Neural sources involved in auditory target detection and novelty processing: an event-related fMRI study. Psychophysiology 38, $133-142$.

Koelsch, S., 2005. Neural substrates of processing syntax and semantics in music. Curr. Opin. Neurobiol. 15, 207-212.

Koelsch, S., Gunter, T., Friederici, A.D., Schroger, E., 2000. Brain indices of music processing: "nonmusicians" are musical. J. Cogn. Neurosci. 12 (3), 520-541.

Koelsch, S., Gunter, T.C., von Cramon, D.Y., Zysset, S., Lohmann, G., Friederici, A.D., 2002. Bach speaks: a cortical "language-network" serves the processing of music. NeuroImage 17 (2), 956-966.

Koelsch, S., Fritz, T., Schulze, K., Alsop, D., Schlaug, G., 2005. Adults and children processing music: an fMRI study. NeuroImage 25, $1068-1076$.

Krumhansl, C.L., 1990. Cognitive Foundations of Musical Pitch. Oxford Univ. Press, New York.

Krumhansl, C.L., 2003. Experimental strategies for understanding the role of experience in music cognition. Ann. N. Y. Acad. Sci. 999, 414-428.

Lerdahl, F., Jackendoff, R., 1983. A Generative Theory of Tonal Music. The MIT Press, Cambridge.

Lewis, P.A., Critchley, H.D., Smith, A.P., Dolan, R.J., 2005. Brain mechanisms for mood congruent memory facilitation. NeuroImage 25, 1214-1223.

Liegeois-Chauvel, C., de Graaf, J.B., Laguitton, V., Chauvel, P., 1999. Specialization of left auditory cortex for speech perception in man depends on temporal coding. Cereb. Cortex 9, 484-496.

Linden, D.E.J., Prvulovic, D., Formisano, E., Vollinger, M., Zanella, F.E., Goebel, R., et al., 1999. The functional neuroanatomy of target detection: an fMRI study of visual and auditory oddball tasks. Cereb. Cortex 9, 815-823.

Lohmann, G., Mueller, K., Bosch, V., Mentzel, H., Hessler, S., Chen, C.K., et al., 2001. LIPSIA-A new software system for the evaluation of functional magnetic resonance images of the human brain. Comput. Med. Imaging Graph. 25, 449-457.

Maccotta, L., Buckner, R.L., 2004. Evidence for neural effects of repetition that directly correlate with behavioral priming. J. Cogn. Neurosci. 16, $1625-1632$.

Maess, B., Koelsch, S., Gunter, T.C., Friederici, A.D., 2001. Musical syntax is processed in Broca's area: an MEG study. Nat. Neurosci. 4 (5), 540-545.

Markowitsch, H.J., Vandekerckhovel, M.M., Lanfermann, H., Russ, M.O., 2003. Engagement of lateral and medial prefrontal areas in the ecphory of sad and happy autobiographical memories. Cortex $39,643-665$.

Mesulam, M.M., Mufson, E.J., 1993. The insula of Reil in man and monkey. In: Jones, E.G., Peters, A. (Eds.), Cerebral Cortex. Association and Auditory Cortices, vol. 4. Plenum Press, New York, p. 179-225.

Meyer, L.B., 1956. Emotion and Meaning in Music. University of Chicago Press, Chicago.

Meyer, M., Friederici, A.D., von Cramon, D.Y., 2000. Neurocognition of auditory sentence comprehension: event related fMRI reveals sensitivity to syntactic violations and task demands. Cogn. Brain Res. 9 (1), 19-33.

Müller, R.-A., Kleinhans, N., Courchesne, E., 2001. Broca's area and the discrimination of frequency transitions: a functional MRI study. Brain Lang. 76, 70-76.

Neumann, J., Lohmann, G., 2003. Bayesian second-level analysis of functional magnetic resonance images. NeuroImage 20, 1346-1355.

Nobre, A.C., Coull, J.T., Frith, C.D., Mesulam, M.M., 1999. Orbitofrontal cortex is activated during breaches of expectations in tasks of visual attention. Nat. Neurosci. 2, 11-12.

Patel, A.D., 2003. Language, music, syntax and the brain. Nat. Neurosci. 6 (7), 674-681.

Patel, A.D., Gibson, E., Ratner, J., Besson, M., Holcomb, P.J., 1998. Processing syntactic relations in language and music: an event-related potential study. J. Cogn. Neurosci. 10 (6), 717-733.

Paulesu, F., Frith, C.D., Frackowiak, R.S.J., 1993. The neural correlates of the verbal component of working memory. Nature 362, 342-345.

Petersson, K.M., Forkstam, C., Ingvar, M., 2004. Artificial syntactic violations activate Broca's region. Cogn. Sci. 2, 383-407.

Platel, H., Price, C.J., Baron, J.C., Wise, R., Lambert, J., Frackowiak, R.S.J., et al., 1997. The structural components of music perception: a functional anatomical study. Brain 120, 229-243.

Poldrack, R.A., Wagner, A.D., Prull, M.W., Desmond, J.E., Glover, G.H., Gabrieli, J.D., 1999. Functional specialization for semantic and phonological processing in the left inferior prefrontal cortex. NeuroImage 10 (1), 15-35.

Pugh, K.R., Shaywitz, B.A., Fulbright, R.K., Byrd, D., Skudlarski, P., Katz, 
L., et al., 1996. Auditory selective attention: an fMRI investigation. NeuroImage 4, 159-173.

Rao, S.M., Mayer, A.R., Harrington, D.L., 2001. The evolution of brain activation during temporal processing. Nat. Neurosci. 4, $317-323$.

Satoh, M., Takeda, K., Nagata, K., Hatazawa, J., Kuzuhara, S., 2001. Activated brain regions in musicians during an ensemble: a PET study. Brain Res. Cogn. Brain Res. 12, 101-108.

Schubotz, R.I., von Cramon, D.Y., 2001a. Functional organization of the lateral premotor cortex: fMRI reveals different regions activated by anticipation of object properties, location and speed. Cogn. Brain Res. $11,97-112$.

Schubotz, R.I., von Cramon, D.Y., 2001b. Interval and ordinal properties of sequences are associated with distinct premotor areas. Cereb. Cortex 11, $210-222$.

Schubotz, R.I., Friederici, A.D., von Cramon, D.Y., 2000. Time percepion and motor timing: a common cortical and subcortical basis revealed by fMRI. NeuroImage 11, 1-12.

Scott, S.K., Blank, S.C., Rosen, S., Wise, R.J.S., 2000. Identification of a pathway for intelligible speech in the left temporal lobe. Brain 123, $2400-2406$.

Talairach, J., Tournoux, P., 1988. Coplanar Stereotaxic Atlas of the Human Brain. Thieme, New York, NY.

Tekman, H.G., Bharucha, J.J., 1998. Implicit knowledge versus psychoacoustic similarity in priming of chords. J. Exp. Psychol. Hum. Percept. Perform. 24 (1), 252-260.

Terhardt, E., 1984. The concept of musical consonance: a link between music and psychoacoustics. Music Percept. 1, 276-295.

Thirion, J.P., 1998. Image matching as a diffusion process: an analogy with Maxell's demons. Med. Image Anal. 2, 243-260.
Tillmann, B., Bigand, E., Pineau, M., 1998. Effects of global and local contexts on harmonic expectancy. Music Percept. 16 (1), 99-117.

Tillmann, B., Bharucha, J.J., Bigand, E., 2000. Implicit learning of tonality: a self-organizing approach. Psychol. Rev. 107 (4), 885-913.

Tillmann, B., Janata, P., Bharucha, J.J., 2003. Activation of the inferior frontal cortex in musical priming. Cogn. Brain Res. 16 (2), 145-161.

Tillmann, B., Bigand, E., Escoffier, N., Lalitte, P., 2006. Influence of harmonic context on musical timbre processing. Eur. J. Cogn. Psychol $18,343-358$.

Ullmann, M.T., 2001. A neurocognitive perspective on language: the declarative/procedural model. Nat. Rev., Neurosci. 2, 717-726.

Wagner, A.D., Koustaal, W., Maril, A., Schacter, D.L., Buckner, R.L., 2000. Task-specific repetition priming in left inferior prefrontal cortex. Cereb. Cortex 10, 1176-1184.

Wise, R.J.S., Scott, S.K., Blank, S.C., Mummery, C.J., Murphy, K., Warburton, E.A., 2001. Separate neural subsystem within 'Wernicke's area'. Brain 124, 83-95.

Wong, P.C.M., Parsons, L.M., Martinez, M., Diehl, R.L., 2004. The role of the insular cortex in pitch pattern perception: the effect of linguistic contexts. J. Neurosci. 24, 9153-9160.

Worsley, K.J., Friston, K.J., 1995. Analysis of fMRI time-series revisitedAgain. NeuroImage 2, 173-181.

Zald, D.H., Kim, S.W., 1996. Anatomy and function of the orbital frontal cortex I: anatomy, neurocircuitry, and obsessive-compulsive disorder. J. Neuropsychiatry Clin. Neurosci. 8, 125-138.

Zatorre, R.J., Evans, A.C., Meyer, E., 1994. Neural mechanisms underlying melodic perception and memory for pitch. J. Neurosci. 14, 1908-1919.

Zatorre, R.J., Mondor, T.A., Evans, A.C., 1999. Auditory attention to space and frequency activates similar cerebral systems. NeuroImage 10 (5), $544-554$. 